\title{
COVERING THE TRACK OF AN ISOTOPY
}

\section{P. ROURKE}

We work in the Polyhedral Category as defined in [3], which consists of polyhedra and polymaps. A polyhedron is a space with a maximal nonempty family of p.l. related triangulations and a polymap is p.l. w.r.t. these triangulations.

An ambient isotopy of a polyhedron $X$ is a level-preserving homeo,

$H: X \times I \rightarrow X \times I$ with $H \mid X \times 1=1$,

$H_{t}: X \rightarrow X$ for $t \in I$ is the homeo induced by $H \mid X \times t$.

An isotopy of $Y$ in $X$ is a level-preserving embedding,

$F: Y \times I \rightarrow X \times I$,

$F_{t}: Y \rightarrow X$ for $t \in I$ is the embedding induced by $F \mid Y \times t$.

We say that $H$ covers $F$ if $H_{t} F_{0}=F_{t}$ for all $t \in I$, and we say that $H$ covers the track of $F$ if $H_{t} F_{0} Y=F_{t} Y$ for all $t \in I$.

Given an isotopy $F$ of $Y$ in $X$, it is not true that there is always an ambient isotopy of $X$ which covers $F$ or even just the track of $F$. For example, if we restrict attention to manifolds and proper embeddings, then classical knots of $S^{1}$ in $S^{3}$ are isotopic embeddings which are not ambient isotopic.

Ambient isotopy is rather more useful than isotopy, but isotopies are usually easier to construct. The following problem is therefore of interest.

Problem A. Given an isotopy $F$ of $Y$ in $X$, under what conditions can we cover $F$ by an ambient isotopy of $X$ ?

For some purposes it is enough to cover the track of $F$, e.g. when considering knots as subspaces rather than embeddings, i.e. working set-wise rather than point-wise; so we have the weaker problem.

Problem B. Under what conditions can we cover the track of an isotopy of $Y$ in $X$ ?

In [1] Zeeman and Hudson give a solution to $A$ for proper embeddings of manifolds. Their condition for coverability is local-unknotting of $F \mid Y \times J$ for any subinterval $J \subset I$, which is always true in codimension $\geqq 3$, see [1] for the precise definition.

In this paper we will be concerned only with Problem B and our main result is that any locally collarable isotopy (definition below) can be track-covered.

Local collarability is a necessary condition for track-covering and, in the case of proper embeddings of manifolds, is strictly weaker than local unknotting.

Received by the editors February 11, 1966. 
The proof of our result rests heavily on techniques of Zeeman [2] and Hudson and Zeeman [1]; however it improves on known answers to Problem B, since it works for any compact polyhedra (not merely for manifolds).

1. Collaring and local collarability. A pair $\{X\}$ consists of a polyhedron $X$ with subpolyhedron $X_{1} \subset X . C(\{X\})$ denotes the cone (pair) on $\{X\}$.

Collaring. Let $\{Y\} \subset\{X\}$ be a subpair (i.e. $Y \subset X$ and $Y_{1} \subset X_{1}$ ), $\{Y\}$ is said to be collared or collarable in $\{X\}$ if $\exists$ a homeo $h:\{M\}$ $\rightarrow\{X\}$ with $h \mid(\{Y\} \times 0)=$ canon. proj. $\{Y\} \times 0 \rightarrow\{Y\}$, where $\{M\}$ denotes the mapping cylinder of $\{Y\} \subset\{X\}$ i.e. $\{M\}=\{Y\}$

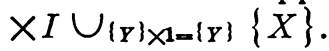

Local Collaring. Let $\{Y\} \subset\{X\}$ and let $P$ be a point in $Y,\{Y\}$ is said to be locally collared in $\{X\}$ at $P$ if $\operatorname{lk}(P,\{X\}) \cong C(\operatorname{lk}(P,\{Y\}))$, as pairs, in any (and $\therefore$ every) triangulation with $P$ a vertex, where the homeo restricts to 1 on $\operatorname{lk}(P,\{Y\})$.

$\{Y\}$ is said to be locally collarable in $\{X\}$ if it is locally collared at each point in $Y$. We have

Collaring Theorem. Let $\{Y\} \subset\{X\}$ be both compact (i.e. $X, X_{1}$, $Y, Y_{1}$ all compact), then $\{Y\}$ is collarable in $\{X\} \Leftrightarrow$ it is locally collarable in $X$.

REMARK. If $\{X\}$ is a properly embedded manifold pair, and $\{Y\}$ is the boundary pair, then local unknotting on the boundary in the sense of $[2] \Rightarrow$ local collarability (straight from the definitions).

However in this case local collarability is a strictly weaker condition, e.g. take $S^{2} \subset S^{4}$ locally knotted somewhere, say at a point $\mathrm{t}_{3} Q$, and define $M=S^{4} \times I$ and $M_{1}=S^{2} \times I \subset M$, then the pair $\{M\}$ $=M_{1} \subset M$ is collarable on its boundary and hence locally collarable but it is locally knotted on the boundary at $Q \times 0$ and $Q \times 1$.

Our Collaring Theorem therefore improves directly on Zeeman's [2] (for locally unknotted properly embedded manifold pairs), the proof however is almost identical.

Proof of the Collaring Theorem. $\Rightarrow$ is trivial, so we just prove $\Leftarrow$. Triangulate $\{X\}$ and $\{Y\}$ by complexes $\{K\},\{L\}$, i.e.

$$
\begin{aligned}
& L \subset K \\
& \cup \quad \cup \\
& L_{1} \subset K_{1},
\end{aligned}
$$

all simplicial, and let $\left\{K^{\prime}\right\},\left\{L^{\prime}\right\}$ denote barycentric first deriveds. 
Order the simplexes of $L$ in order of increasing dimension as $A_{1}, A_{2}$, $\cdots, A_{t}$. Denote the barycentre of $A_{i}$ by $P_{i}$ for each $i$, and define the dual (complex) of $A_{i}$ in $L^{\prime}$ for each $i$ as follows (denoted by $A_{i}^{*}$ ),

$$
A_{i}^{*}=\bigcap_{P \text { a vertex of } A_{i}} \bar{s} t\left(P, L^{\prime}\right) .
$$

$A_{i}^{*}$ is a cone vertex $P_{i}$ for each $i$, and we write the base $A_{i}^{\sim}$, so $A_{i}^{*}$ $=P_{i} \cdot A_{i}^{\sim}$.

Write $\left\{A_{i}\right\}$ etc. for the corresponding subpair of $\{Y\}$ to $A_{i}$ etc., i.e. $\left\{A_{i}\right\}=A_{i} \cap Y_{1} \subset A_{i}$. Let $\{M\}$ be the mapping cylinder of $\{Y\} \subset\{X\}$ (defined above) and using the linear structure of the prisms $A_{j} \times I$ define, for each $i$, the join $A_{i}^{+}=A_{i} \times 0 \cdot A_{i}^{*} \times 1$, and define inductively

$$
\begin{gathered}
M^{0}=X, \quad Y^{0}=Y, \quad M^{i}=M^{i-1} \cup A_{i}^{+}, \\
Y^{i}=\left(Y^{i-1}-\partial A_{i} \times 0 \cdot A_{i}^{*} \times 1\right) \cup A_{i} \times 0 \cdot A_{i} \times 1 ;
\end{gathered}
$$

loosely think of $Y^{i}$ as obtained from $Y^{i-1}$ by moving $P_{i} \times 1$ up to $P_{i} \times 0$.

Note that $M^{t}=M$ and $Y^{t}=Y \times 0$; denote by $\left\{A_{i}^{+}\right\}\left\{M^{i}\right\},\left\{Y^{i}\right\}$ the corresponding subpairs of $\{M\}$.

Now there is a natural homeo $\pi_{i}:\left\{Y^{i}\right\} \rightarrow\{Y\}$ for each $i$ induced by projection $\{Y\} \times I \rightarrow\{Y\}$ (think of it as moving the $P_{j} \times 0$ back down to $P_{j} \times 1$ in reverse order); note that $\pi_{0}=1$.

We will define homeos $h_{i}:\left\{M^{i}\right\} \rightarrow\{X\}$ for each $i$ such that $h_{i} \mid\left\{Y^{i}\right\}$ $=\pi_{i}$, and this is by induction on $i$ starting with $h_{0}=1 . h_{t}:\{M\} \rightarrow\{X\}$ will be the required homeo for the definition of collaring since $h_{t}\left|(\{Y\} \times 0)=\pi_{t}\right|(\{Y\} \times 0)=$ projection.

So suppose $h_{i-1}$ to be defined as above and define $h_{i}$. Note that

$$
\begin{gathered}
M^{i}=M^{i-1} \cup A_{i}^{+}=M^{i-1} \cup\left(P_{i} \times 0 \cdot \partial A_{i} \times 0 \cdot A_{i}^{*} \times 1\right) ; \\
h_{i-1}\left(\partial A_{i} \times 0 \cdot A_{i}^{*} \times 1\right)=\pi_{i-1}\left(\partial A_{i} \times 0 \cdot A_{i}^{*} \times 1\right)=\bar{s} t\left(P_{i}, L^{\prime}\right) .
\end{gathered}
$$

Therefore $h_{i-1}$ and conical extension (vertex $\left.P_{i} \times 0\right)$ gives a homeo

$$
\bar{h}_{i-1}:\left\{M^{i}\right\} \rightarrow\{X\} \cup P_{i} \times 0 \cdot \bar{s} t\left(P_{i},\left\{L^{\prime}\right\}\right) .
$$

Now

$$
\bar{s} t\left(P_{i},\left\{K^{\prime}\right\}\right)=P_{i} \cdot \operatorname{lk}\left(P_{i},\left\{K^{\prime}\right\}\right)=P_{i} \cdot C\left(\operatorname{lk}\left(P_{i},\left\{L^{\prime}\right\}\right)\right)
$$

by local collarability. Let the vertex of $C\left(\operatorname{lk}\left(P_{i},\left\{L^{\prime}\right\}\right)\right)$ be $Q_{i} ; P_{i}$. $C\left(1 \mathrm{k}\left(P_{i},\left\{L^{\prime}\right\}\right)\right)$ can be thought of as a cone pair vertex at some point $T_{i}$ on the ray $Q_{i} P_{i}$, and this represents $\bar{s} t\left(P_{i},\left\{K^{\prime}\right\}\right)$ as $T_{i} \cdot \Sigma \operatorname{lk}\left(P_{i},\left\{L^{\prime}\right\}\right)[\Sigma$ denotes suspension].

But $P_{i} \times 0 \cdot \bar{s} t\left(P_{i},\left\{L^{\prime}\right\}\right) \cup \bar{s} t\left(P_{i},\left\{K^{\prime}\right\}\right) \cong P_{i} \cdot \Sigma \operatorname{lk}\left(P_{i},\left\{L^{\prime}\right\}\right)$. So we 


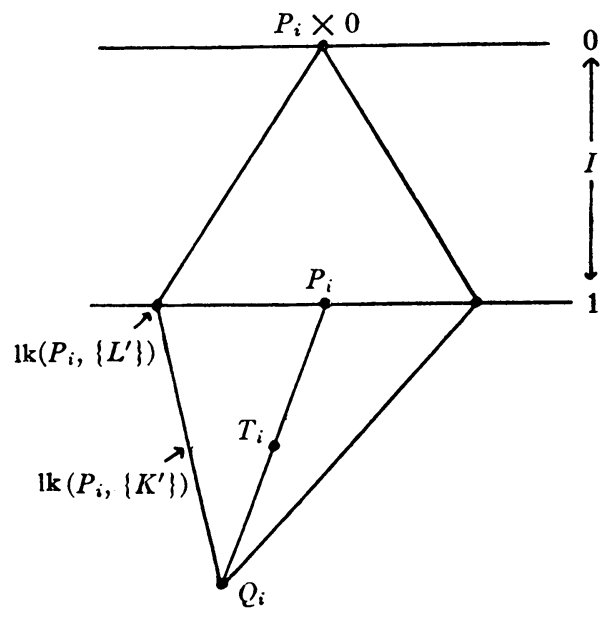

can define a homeo $\beta_{i}: P_{i} \times 0 \cdot \bar{s} t\left(P_{i},\left\{L^{\prime}\right\}\right) \cup \bar{s} t\left(P_{i},\left\{K^{\prime}\right\}\right) \rightarrow \bar{s} t\left(P_{i},\left\{K^{\prime}\right\}\right)$ as follows: map $P_{i} \times 0 \cdot \operatorname{lk}\left(P_{i},\left\{L^{\prime}\right\}\right)$ conically to $P_{i} \cdot \operatorname{lk}\left(P_{i},\left\{L^{\prime}\right\}\right)$, map $\operatorname{lk}\left(P_{i},\left\{K^{\prime}\right\}\right)$ by the identity, and extend to the rest conically, vertices $P_{i}$ and $T_{i}$ respectively. Extend $\beta_{i}$ by the identity on $\{X\}-\bar{s} t\left(P_{i},\left\{K^{\prime}\right\}\right)$, and define $h_{i}=\beta_{i} \bar{h}_{i-1}$ and the required condition is just by the construction of $\beta_{i}$.

This completes the Collaring Theorem.

Addenda. This section works just as well for flags, i.e. sequences $\{X\}=X_{n} \subset X_{n-1} \subset \cdots \subset X_{1} \subset X$, without alteration.

The Collaring Theorem also works with some of the compactness conditions relaxed (we leave the reader to investigate if interested).

2. Covering the track of an isotopy. An isotopy $F: Y \times I \rightarrow X \times I$ is said to be locally collarable if, for each subinterval $J \subset I$, the pair $F(Y \times \partial J), X \times \partial J$ is locally collarable in $F(Y \times J), X \times J(\partial J$ denotes the boundary of $J$ ). Our main result is

Track Covering Isotopy Theorem. Let $X, Y$ be compact and $F$ an isotopy of $Y$ in $X$.

$\exists$ an ambient isotopy of $X$ covering the track of $F$;

$\Leftrightarrow F$ is locally collarable.

REMARK. As in the Collaring Theorem, in the case of proper embeddings of manifolds, local collarability is strictly weaker than local unknotting.

Proof of The TheOREM $\Rightarrow$ an ambient isotopy collars $F(Y \times \partial J)$, $X \times \partial J$ in $F(Y \times J), X \times J$ for each $J \subset I$, and therefore implies local collarability. $\Leftarrow$ comes quickly from two of the techniques of Hudson and Zeeman [1], as follows: 
Proposition. There is a short ambient isotopy of $X$ which covers the track of the beginning of $F$.

Proof. By local collarability and the Collaring Theorem, the pair $F(Y \times 0), X \times 0$ is collarable in $F(Y \times I), X \times I$.

This implies that $\exists$ an embedding, $c: X \times I, \quad F_{0} Y \times I \rightarrow X \times I$, $F(Y \times I)$ which maps $X \times I$ onto a neighborhood of $X \times 0$ in $X \times I$.

Now by $\left[1\right.$, Lemma 5] we can change $c$ to $c^{*}$ with $c^{*}$ level preserving in $[0, \delta]$; the method of proof is to $\operatorname{star} c$ on the $\delta$ level for all simplexes meeting the $\delta$ level, for $\delta$ smaller than all meshes in some triangulation of $c$.

Note therefore that $c^{*}(X \times[0, \delta])$ is still a neighborhood of $X \times 0$ in $X \times I$ and that $c^{*}\left(F_{0} Y \times I\right)$ is still $\subset F(Y \times I)$.

$c^{*}\left(c_{0}^{*-1} \times 1\right) \mid[0, \delta]$ is therefore a short ambient isotopy of $X$ covering the beginning of the track of $F$, proving the proposition.

Now by the proposition and the fact that local collarability gives the same condition on either side of each level, we get, for each $t \in I$, a short "ambient isotopy" $H^{t}$ of $X$, which covers the track of $F$ in a neighborhood of $t$.

The theorem therefore follows by the argument in $[1$, proof of Theorem 2], which is a compactness argument. By compactness of $I$, we get a set of abutting intervals covering $I$ with "short ambient isotopies" on each covering of the track of $F$; we form $H$ by piecing them together, taking care to make them agree where they meet-see [1] for details.

Addenda. (1) We can choose $H$ to keep fixed the complement of a neighborhood of the track of $F$ in $X\left(=\bigcup_{t} F_{t} Y \subset X\right)$.

This is because in the Collaring Theorem, as applied in the above proof, we can choose our collar to be standard outside a neighborhood of $F(Y \times 0)$, which gives the short ambient isotopy to be the identity outside a neighborhood of $F_{0} Y$.

(2) Some of the compactness conditions can be relaxed in the theorem (e.g. by (1) it is enough for a neighborhood of the track of $F$ to be compact).

\section{REFERENCES}

1. J. F. P. Hudson and E. C. Zeeman, On combinatorial isotopy, Inst. Hautes Études Sci. Publ. Math. 19 (1964), 69-94. 526.

2. E. C. Zeeman, Unknotting combinatorial balls, Ann. of Math. 78 (1963), 501-

3. - Seminar on combinatorial topology, Inst. Hautes Études Sci., Paris, 1963-1965.

Queen Mary College, London, England 